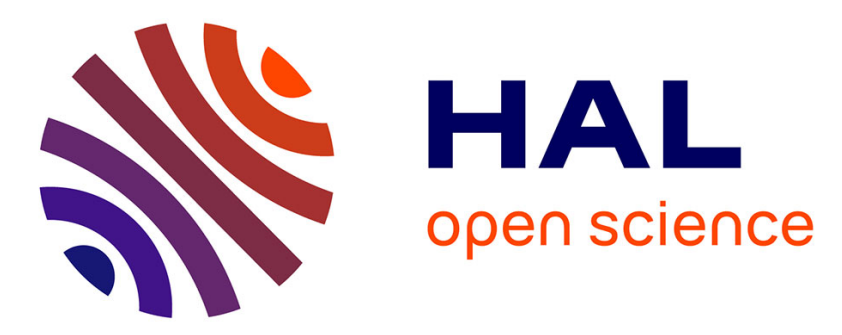

\title{
Energy-efficient time-triggered communication policies for wireless networked control systems
}

Vineeth Varma, André M de Oliveira, Romain Postoyan, Irinel-Constantin

Morarescu, Jamal Daafouz

\section{- To cite this version:}

Vineeth Varma, André M de Oliveira, Romain Postoyan, Irinel-Constantin Morarescu, Jamal Daafouz. Energy-efficient time-triggered communication policies for wireless networked control systems. IEEE Transactions on Automatic Control, 2020, 65 (10), pp.4324-4331. 10.1109/TAC.2019.2953816 . hal02361427

\section{HAL Id: hal-02361427 \\ https://hal.science/hal-02361427}

Submitted on 13 Nov 2019

HAL is a multi-disciplinary open access archive for the deposit and dissemination of scientific research documents, whether they are published or not. The documents may come from teaching and research institutions in France or abroad, or from public or private research centers.
L'archive ouverte pluridisciplinaire HAL, est destinée au dépôt et à la diffusion de documents scientifiques de niveau recherche, publiés ou non, émanant des établissements d'enseignement et de recherche français ou étrangers, des laboratoires publics ou privés. 


\title{
Energy-efficient time-triggered communication policies for wireless networked control systems
}

\author{
Vineeth S. Varma, André M. de Oliveira, Romain Postoyan, Irinel-Constantin Morărescu and Jamal Daafouz
}

\begin{abstract}
Energy-efficient communication protocols have become an important topic over the past two decades due to environmental issues, increased monetary costs of energy consumption, and limited battery capacities of sensors and mobile devices. In this context, we present a novel approach to design energy-efficient time-triggered communication policies for wireless networked control systems, while ensuring a given control performance. We consider a plant, modeled as a deterministic discrete-time linear system, which is controlled through a wireless network by an output-feedback law. We proceed by emulation, i.e., we construct the controller to stabilize the origin of the plant while ignoring communication constraints. Next, the wireless network is taken into account and we assume that the probability of packet drops depends on the transmission signal power. We introduce the notion of stochastic allowable transmission interval (SATI) to characterize stabilizing time-triggered transmission policies. We then explain how to minimize the average energy expenditure of the transmitting devices while satisfying the SATI constraints, thus ensuring the control requirements. Simulations results are provided to illustrate the trade-off between the communication and the control costs.
\end{abstract}

\section{INTRODUCTION}

Energy-efficiency in communication systems has gained a rising amount of interest in recent years [1], [2]. For devices such as cellular phones, unplugged laptops, wireless sensors, and mobile robots, smart management of energy is essential due to the limited supply of energy available. Various studies have investigated the design of energy efficient communication systems, i.e., minimizing energy while maintaining a certain quality of service parameter, or maximizing the ratio of data rate to energy consumed, see [2] for an extensive survey. When the wireless communication is with single antennas, one of the most relevant techniques to improve energy efficiency is that of transmission power control. In works like [3], [4], transmission power is optimized so that the ratio between the number of packets transmitted successfully to the power consumed is maximized.

In the context of control systems, wireless networks offer appealing features as they allow remote control and exhibit many advantages over traditional wired point-to-point set-ups in terms of flexibility, ease of maintenance, reduced weight,

V. S. Varma, R. Postoyan, I. C. Morărescu and J. Daafouz are with the Université de Lorraine, CNRS, CRAN, F-54000 Nancy, France, vineeth. satheeskumar-varma@univ-lorraine.fr.

A. M. Oliveira is with the Departamento de Engenharia de Telecomunicações e Controle, Escola Politécnica da Universidade de São Paulo, CEP 05508-010, São Paulo, Brazil, and is a visiting PhD student at CRAN.

This work was partially funded by the ANR via grant HANDY, number ANR-18-CE40-0010, and by the São Paulo Research Foundation, grant FAPESP-2017/06358-5. and volume. On the other hand, the communication constraints induced by the network need to be appropriately handled to guarantee the desired performance for the closed-loop system, which motivates research on so-called networked control systems (NCS), see e.g. [5] and the references therein. Often, the energy expenditure due to communications is ignored in works on NCS, or it is treated in an ad-hoc way, in the sense that the less often we transmit (or the smaller the packet length), the less energy we spend to communicate for instance. In wireless communication, transmitting less often does not always imply improving the energy efficiency of communication as the probability of successful communication depends on several factors like the power of the transmission signal, the quality of the wireless channel, noise levels, etc. [1], [6].

A few researchers have recently published results, which consider the above mentioned problem. The paper [7] provides a list of works that implement energy-efficient communication design in the context of NCS, which mostly concentrates on estimation, as in [8], or proposed numerical heuristics, as in [9]. On the other hand, the authors of [10] and [11] studied the problem of minimization of a cost defined as the sum of the control cost and the wireless power and proposed eventtriggered transmission strategies. There are also works like [12], which deal with power control and energy efficiency but for the purpose of filtering, and not for stability and control performance as is done in our work.

In this paper, we consider plants modeled as deterministic linear time-invariant systems and we design time-triggered energy-efficient communication policies in the sense that the transmission strategy depends on the elapsed time since the last successful communication. We are motivated by applications involving sensor nodes with limited computational and energy capacities. In this context, while the proposed policy may generate more transmissions than event-triggered control as in [11], it exhibits various advantages such as ease of implementation and relaxed information requirements at the sensor. For example, in the time-triggered framework, if the sensor is a camera, it does not have to process the images captured in order to evaluate the triggering condition and decide when to transmit the images.

This motivates us to develop a new notion, referred to as, the stochastic allowable transmission interval (SATI). The SATI is described by a certain interval length $N$, a cumulative probability $\eta$ of a transmission to occur before $N$ steps have elapsed since the previous successful transmission, and $\delta$ the instantaneous transmission success probability after $N$ steps have passed. Here, $\delta$ corresponds to the success probability when using the maximum possible transmission power. This 
approach can be seen as a stochastic version of time-triggered control instead of independently distributed probabilities of the inter-transmissions intervals as in [13], [14]. During the $N$ steps, packet drop probabilities can be designed in a flexible manner by adapting the transmission power as long as the constraint on $\eta$ is satisfied. This flexibility can be exploited in order to design energy efficient communication policies. After $N$ steps have passed, the maximum power is used until a successful transmission occurs. Compared to [11], (i) we design time-triggered policies, as opposed to event-triggered policies, which is a different implementation paradigm with its associated pros and cons as explained above, (ii) we consider output feedback laws, and not only statefeedbacks, (iii) we allow for varying holding strategies not only model-based ones, and (iv) our approach allows minimization of the communication cost while ensuring a given control performance. We started to address this problem in [6] using a deterministic constraint on the maximum allowed time between two transmissions, which resulted in very strong assumptions of the channel state, in particular, we assumed that a successful transmission is possible at any given time. This condition is no longer needed in this work. Compared to [15], where we introduce the notion of SATI, we now account for control performance costs in addition to results on stability and also perform the optimization of communication energy in this framework.

Notation. Let $\mathbb{R}:=(-\infty, \infty), \mathbb{R}_{\geq 0}:=[0, \infty)$, and $\mathbb{R}_{>0}:=$ $\mathbb{R}_{\geq 0} \backslash\{0\}$, as well as $\mathbb{Z}_{\geq 0}:=\{0,1,2, \ldots\}$ and $\mathbb{Z}_{>0}:=$ $\mathbb{Z}_{\geq 0} \backslash\{0\}$. We use Pr for the probability and $\mathbb{E}$ for the expectation taken over the relevant stochastic variables, and $\mathbf{1}_{S}(x)$ for the indicator function, taking the value 1 when $x \in S$ and 0 otherwise.

\section{PRoblem StATEMENT}

\section{A. Setup}

We consider the discrete-time linear system

$$
\begin{aligned}
x_{p}(t+1) & =A_{p} x_{p}(t)+B_{p} u(t) \\
y(t) & =C_{p} x_{p}(t),
\end{aligned}
$$

where $t \in \mathbb{Z}_{\geq 0}$ is the time, $x_{p}(t) \in \mathbb{R}^{n_{x}}$ is the plant state, $u(t) \in \mathbb{R}^{n_{u}}$ is the control input, $y(t) \in \mathbb{R}^{n_{y}}$ is the output and $n_{x}, n_{u}, n_{y} \in \mathbb{Z}_{>0}$. The pairs $\left(A_{p}, B_{p}\right)$ and $\left(A_{p}, C_{p}\right)$ are assumed to be stabilizable and detectable, respectively. We proceed by emulation and we first construct a stabilizing controller for system (1) given by

$$
\begin{aligned}
x_{c}(t+1) & =A_{c} x_{c}(t)+B_{c} y(t) \\
u(t) & =C_{c} x_{c}(t)+D_{c} y(t),
\end{aligned}
$$

where $x_{c}(t) \in \mathbb{R}^{n_{x}}$ is the controller state. Such a stabilizing controller always exists since $\left(A_{p}, B_{p}\right)$ is stabilizable and $\left(A_{p}, C_{p}\right)$ is detectable.

We are interested in the scenario where plant (1) and controller (2) communicate over a wireless channel as illustrated in Figure $1^{1}$. As a result, the feedback loop is no longer

\footnotetext{
${ }^{1}$ The forthcoming results can be extended to the case where the network is used to communicate both the sensors data and the control input, at the price of more technicalities. We do not address it in this publication to not blur our main message.
}

closed at every time instant $t \in \mathbb{Z}_{>0}$, but only at the instants $t_{i}, i \in \mathbb{Z}_{>0}$, when communication is successful. Note that we ignore delays and quantization effects, but an alternate network configuration or the effect of delays can be easily treated as explained later in Remark 1.

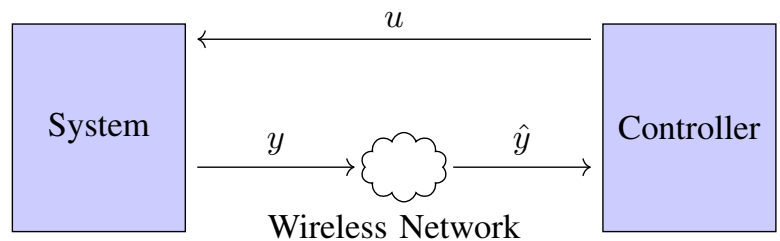

Fig. 1: Schematic of the networked control system.

When the wireless network is present as shown in Fig. 1, system (1) remains unchanged, but (2) becomes

$$
\begin{aligned}
x_{c}(t+1) & =A_{c} x_{c}(t)+B_{c} \hat{y}(t) \\
u(t) & =C_{c} x_{c}(t)+D_{c} \hat{y}(t),
\end{aligned}
$$

where $\hat{y}(t)$ denotes the networked version of the output $y(t)$.

Between two successive successful transmission instants, $\hat{y}$ is held using general holding functions of the form

$$
\hat{y}(t)=C_{g} \hat{y}(t-1)+D_{g} x_{c}(t)
$$

when $t \in \mathbb{Z}_{\geq 0} \backslash\left\{t_{i}\right\}_{i \in \mathbb{Z}_{>0}}$. For instance, zero-order-hold devices lead to $\left(C_{g}, D_{g}\right)=(I, 0)$, and the zeroing strategy [16] corresponds to $\left(C_{g}, D_{g}\right)=(0,0)$. When the $x_{c}$-system in (2) is a Luenberger observer, we can also use a model-based holding strategy like in [13], which gives

$$
\hat{y}(t)=C_{p} B_{p} D_{c} \hat{y}(t-1)+C_{p}\left(A_{p}+B_{p} C_{c}\right) x_{c}(t)
$$

that is, $\left(C_{g}, D_{g}\right)=\left(C_{p} B_{p} D_{c}, C_{p}\left(A_{p}+B_{p} C_{c}\right)\right)$. Our results cover all these cases. At $t_{i}, i \in \mathbb{Z}_{>0}$, the controller receives the current value of the plant output, hence $\hat{y}\left(t_{i}\right)=y\left(t_{i}\right)$. We concatenate all the state variables, for any $t \in \mathbb{Z}_{\geq 0}$, as

$$
\chi(t):=\left[\begin{array}{lll}
x_{p}(t)^{T} & x_{c}(t)^{T} & \hat{y}(t-1)^{T}
\end{array}\right]^{T}
$$

with $\hat{y}(-1)$ being chosen arbitrarily in $\mathbb{R}^{n_{y}}$. The overall system is given by

$$
\chi(t+1)=\left\{\begin{array}{l}
\mathcal{A}_{1} \chi(t) \\
\text { if transmission is successful at } t \\
\mathcal{A}_{0} \chi(t) \\
\text { otherwise }
\end{array}\right.
$$

where

$$
\mathcal{A}_{1}:=\left[\begin{array}{ccc}
A_{p}+B_{p} D_{c} C_{p} & B_{p} C_{c} & 0 \\
B_{c} C_{p} & A_{c} & 0 \\
C_{p} & 0 & 0
\end{array}\right]
$$

and

$$
\mathcal{A}_{0}:=\left[\begin{array}{ccc}
A_{p} & B_{p}\left(C_{c}+D_{c} D_{g}\right) & B_{p} D_{c} C_{g} \\
0 & A_{c}+B_{c} D_{g} & B_{c} C_{g} \\
0 & D_{g} & C_{g}
\end{array}\right] .
$$

To complete model (7), we need to formalize the definition of the successful transmission instants $t_{i}$ 's, and thus to describe the wireless network in more detail: this is the purpose of the 
next subsection.

Remark 1: Note that model (7) can also be easily adapted to situations where the wireless network is between the communication between the controller and the plant actuators instead of with the sensor as explained in [15]. Additionally, when the network has a fixed known delay, the framework of this paper can be easily extended by augmenting $\chi(t)$ to keep track of the delayed versions of the state without any change in the analysis presented in the remaining part of this paper. The model can also be easily adapted to the case.

\section{B. Wireless network}

Plant sensors are associated to a node, which is connected to the wireless network. This node sends packet containing the current measurements to the controller using a power $p(t) \in\left[0, p_{\max }\right]$ at time $t \in \mathbb{Z}_{\geq 0}$, with $p_{\max }>0$ denoting the maximum power at which the transmitting antenna can operate in. We assume in the first place that the probability that a packet is received by the controller at time $t \in \mathbb{Z}_{\geq 0}$ depends on the transmission power $p(t)$ as follows.

Assumption 1: The probability of a packet to be dropped when the transmitting antenna uses a power of $p(t)$ at time $t$ is given by

$$
e(p(t))=\exp (-c p(t))
$$

where $c \in \mathbb{R}_{>0}$ is a constant, which depends on the channel statistics.

Assumption 1 is often used in the communication literature for Rayleigh channel fading as seen in [4], [17] when the actual wireless channel state is not known to the transmitter. We also assume that the sensors know if a transmission was successful via a simple acknowledgment scheme (ACK), which is common in digital communication protocols [1].

Assumption 2: The event of a successful transmission is known at the sensor when the NCS is like in Figure 1 via ACK.

The ACK only requires a few bits and so the energy required to send it in comparison to the sensor measurement (of much larger data size) is negligible. Additionally, we are concerned with the energy expenditure of the sensor node which may have a limited energy capacity while the ACK is sent by the controller which may have a larger energy supply. A consequence of Assumption 1 is that the successful transmission instants $t_{i}, i \in \mathbb{Z}_{>0}$, are stochastic variables [17]. As a result, (7) can be rewritten as, for any $t \in \mathbb{Z}_{\geq 0}$,

$$
\chi(t+1)=\left\{\begin{array}{l}
\mathcal{A}_{1} \chi(t) \text { with probability } 1-e(p(t)), \\
\mathcal{A}_{0 \chi} \chi(t) \text { with probability } e(p(t)) .
\end{array}\right.
$$

The maximum power that can be used by the transmitter is bounded by $p_{\max }$, which implies that the maximum probability for a packet to be successfully received is given by $\delta:=$ $1-e\left(p_{\max }\right)$ according to Assumption 1. Parameter $0<\delta<1$ represents the physical limitations of the communication system in terms of packet success [18]. However transmitting with this success probability will consume a lot of communication resources. This motivates us to design communication schemes that do not always require operating at $p_{\max }$, specifically the SATI as explained in the following.

\section{Stochastic allowable transmission interval}

Time-triggered control results are traditionally based on the MATI in the NCS literature, see e.g. [19], [20], which means that

$$
t_{i+1}-t_{i} \leq N
$$

where $N$ is the so called MATI, for all $i \in \mathbb{Z}_{>0}$. Condition (12) is impossible to ensure under Assumption 1 as the $t_{i}$ 's are stochastic variables. We therefore need a new notion, which should still involve a certain length of the inter-transmission intervals $N$ as in (12), but also the probability that a successful transmission occurs within this interval, and the probability of successful transmission after this interval. We call this new notion $(\eta, \delta)$-SATI. Parameter $\eta \in[0,1]$ is the cumulative probability that communication was successful within $N$ steps since the last successful transmission, and $\delta$ is the probability of successful transmission when the time since the previous transmission is larger than $N$. The parameter $N$ basically denotes a critical limit: if this limit is crossed at some $t$, all the available resources must be used for attempting transmission, i.e., use $p(t)=p_{\max }$ and therefore a transmission success rate of $\delta$.

To formalize the concept of SATI, we first introduce the clock $\tau(t) \in \mathbb{Z}_{\geq 0}$ which counts the number of time instants since the last successful transmission, i.e.

$$
\tau(t+1)=\left\{\begin{array}{lr}
\tau(t)+1 & \text { if transmission failed } \\
1 & \text { otherwise }
\end{array}\right.
$$

Let $N \in \mathbb{Z}_{\geq 0}$ be a given transmission interval length. The time-triggered power control policies we envision are such that $p(t)$ depends on $\tau(t)$. Due to Assumption 2, the sensor always has access to $\tau(t)$ and therefore, this type of policies are always possible. The $(\eta, \delta)$-SATI notion imposes that $p(t)=p_{\max }$ when $\tau(t)>N$, so that the probability of successful transmission after $N$ steps have passed since the last transmission is $\delta$. However, when $\tau(t) \leq N$, we have a certain degree of flexibility in choosing $p(t)$. A time-triggered power control law $\mathbf{p}(\bar{p})$ satisfying the SATI constraints is therefore described using a power control policy vector $\bar{p}$, where $\bar{p} \in\left[0, p_{\max }\right]^{N}$, which implies

$$
p_{\bar{p}}(t)=\left\{\begin{array}{l}
\bar{p}_{n} \\
\text { if } \tau(t)=n \text { and } \tau(t) \leq N \\
p_{\max } \\
\quad \text { if } \tau(t)>N .
\end{array}\right.
$$

Since the probability to transmit within the interval of length $N$ is $\eta, \bar{p}$ must also satisfy the following constraint

$$
1-\prod_{n=1}^{N} e\left(\bar{p}_{n}\right)=\eta \text {. }
$$

We can thus describe $\tau(t)$ with the following stochastic process

$$
\begin{aligned}
& \operatorname{Pr}(\tau(t+1)=n+1 \mid \tau(t)=n, n \in\{1, \ldots, N\})=e\left(\bar{p}_{n}\right), \\
& \operatorname{Pr}(\tau(t+1)=1 \mid \tau(t)=n, n \in\{1, \ldots, N\})=1-e\left(\bar{p}_{n}\right) \\
& \operatorname{Pr}(\tau(t+1)=1 \mid \tau(t)=n, n \in\{N+1, \ldots\})=\delta \\
& \operatorname{Pr}(\tau(t+1)=n+1 \mid \tau(t)=n, n \in\{N+1, \ldots\})=1-\delta .
\end{aligned}
$$


We also define

$$
\widehat{\mathcal{P}}_{N, \eta}:=\left\{\bar{p} \in\left[0, p_{\max }\right]^{N} \mid \prod_{n=1}^{N} e\left(\bar{p}_{n}\right)=1-\eta\right\},
$$

which is the set of policies which satisfy the SATI constraints. If the associated power control law $\mathbf{p}(\bar{p})$ satisfies the desired stability or performance criteria for (11) for any $\bar{p} \in \widehat{\mathcal{P}}_{N, \eta}$, we say that $N$ is an $(\eta, \delta)$-SATI. Now, we proceed to formally state the objectives of this work.

\section{Objectives}

The two main objectives of this work are as follows.

1) Provide conditions on $N, \eta$ and $\delta$ such that we can guarantee mean-square stability (MSS) of (11), according to definition 3.8 in [21]. Moreover, we want to ensure a specified bound on the control performance cost defined for any $\chi(0) \in \mathbb{R}^{2 n_{x}+n_{y}}$ as

$$
J_{\mathrm{CONT}}(\chi(0)):=\sum_{t=0}^{\infty} \mathbb{E}\left[\chi(t)^{T} Q \chi(t)\right],
$$

for a given real, symmetric and positive semi-definite matrix $Q$, and $p(t)$ is given by the SATI rule (14).

2) Minimize the expected average communication energy, see for instance [22], defined as

$$
J_{\mathrm{COMM}}(\bar{p}):=\lim _{T \rightarrow \infty} \frac{\sum_{t=1}^{T} \mathbb{E}\left[p_{\bar{p}}(t)\right]}{T},
$$

when $p_{\bar{p}}(t)$ is given by (14).

The expectation $\mathbb{E}$ in both 1) and 2) is to account for the stochastic nature of packet drops. Note that MSS automatically implies robustness with respect to additive noise in (1)-(2) as shown by Theorem 3.3 in [21]. In the next section, we provide conditions on $\eta, \delta$ and $N$ such that the item 1) is satisfied.

\section{MAIN RESUltS}

\section{A. The Markov jump linear model}

For the purpose of analysis, we first rewrite the model (11) with the power control law $\mathbf{p}(\bar{p}), \bar{p} \in \widehat{\mathcal{P}}_{N, \eta}$ as a Markov jump linear system (MJLS). System (11) is a linear jump system with two modes, depending on whether a successful transmission occurs at time $t \in \mathbb{Z}_{\geq 0}$, but this is not an MJLS as the transition probabilities depend on the power control law. Nevertheless, when the power control law is determined by the policy (14), we can still formulate (11) as an MJLS by artificially increasing the number of modes. When communication is successful, i.e. $\tau(t)=1, \chi(t+1)=\mathcal{A}_{1} \chi(t)$ and $\chi(t+1)=\mathcal{A}_{0} \chi(t)$ otherwise. Therefore, we have a MJLS with 2 distinct dynamics, but $N+1$ virtual modes (the dynamics are identical for $N$ of these modes) as shown in Figure 2. Then, the Markov chain $\theta(t) \in\{1,2, \ldots, N+1\}$ is defined as $\theta(t)=\tau(t)$ when $\tau(t) \leq N$ and $\theta(t)=N+1$ when $\tau(t)>N$. The associated jump probability matrix is $\Pi$ given by

$$
\Pi(\bar{p}):=\left[\begin{array}{ccccc}
1-e\left(\bar{p}_{1}\right) & e\left(\bar{p}_{1}\right) & 0 & \ldots & 0 \\
\vdots & & & & \\
\delta & 0 & 0 & \ldots & 1-\delta
\end{array}\right]
$$

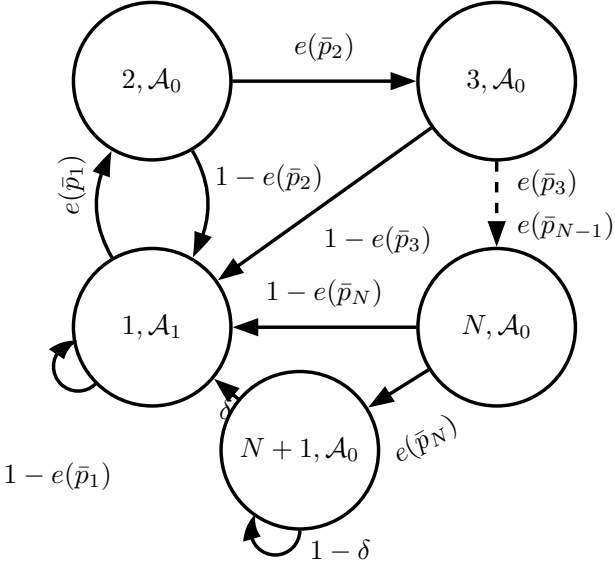

Fig. 2: The Markov chain with the modes representing the states

which follows from Figure 2. There is a probability to jump to mode 1 , i.e., to communicate, given by $1-e\left(\bar{p}_{\theta(t)}\right)$ from any mode $\theta(t)$. The only other mode that can be reached from $\theta(t)$ is one with $\tau(t+1)=\tau(t)+1$, resulting in 0 probability of jumps to all the other states. Finally, it is also possible to jump from $\theta(t)=N+1$ to $\theta(t+1)=N+1$ as $\theta(t)=N+1$ represents any $\tau(t)>N$. As a result, the dynamics (7) are rewritten as

$$
\begin{aligned}
\chi(t+1) & =\mathcal{A}_{\mathbf{1}_{\{1\}}(\theta(t)) \chi(t),} \\
\operatorname{Pr}(\theta(t+1)=j \mid \theta(t)=i) & =\Pi_{i j}(\bar{p}), 1 \leq i, j \leq N+1,
\end{aligned}
$$

for $\bar{p} \in \widehat{\mathcal{P}}_{N, \eta}$.

Our first objective in the following is to provide explicit and computable bounds on the quadratic cost defined as in (18) for a given $\eta, \delta$, intervals $N$, an initial condition $\chi(0)$, and for all $\bar{p} \in \widehat{\mathcal{P}}_{N, \eta}$ while ensuring MSS for (11). This bound must be independent of the actual power control policy $\bar{p}$ as long as $\bar{p} \in \widehat{\mathcal{P}}_{N, \eta}$. For convenience, we define for $N \in \mathbb{Z}_{\geq 0}$,

$$
\bar{Q}_{N}(n):=\sum_{i=n}^{N}\left(\mathcal{A}_{0}^{T}\right)^{i-n} Q \mathcal{A}_{0}^{i-n},
$$

for $n \in\{2, \ldots, N\}$, along with

$$
\bar{P}_{N}(n):=\left(\mathcal{A}_{0}^{T}\right)^{N+1-n} P_{N+1} \mathcal{A}_{0}^{N+1-n}+\eta S+\bar{Q}_{N}(n),
$$

for all $n \in\{2, \ldots, N\}, \bar{P}_{N}(1):=P_{1}$ and $\bar{P}_{N}(N+1):=$ $P_{N+1}$.

\section{B. Stability and performance}

The next theorem provides LMI conditions for obtaining bounds on the cost (18) and ensuring MSS for system (11).

Theorem 1: Given $N \in \mathbb{Z}_{>0}, \eta \in[0,1)$ and $\delta \in[0,1)$, suppose that there exist symmetric positive definite matrices $P_{1}, P_{N+1}$, and $S$ of compatible dimensions such that

$$
\begin{aligned}
P_{1}> & \mathcal{A}_{1}^{T}\left[(1-\eta)\left(\mathcal{A}_{0}^{T}\right)^{N-1} P_{N+1} \mathcal{A}_{0}^{N-1}+\eta S\right] \mathcal{A}_{1} \\
& +\mathcal{A}_{1}^{T} \bar{Q}_{N}(2) \mathcal{A}_{1}+Q, \\
P_{N+1}> & \mathcal{A}_{0}^{T}\left[(1-\delta) P_{N+1}+\delta P_{1}\right] \mathcal{A}_{0}+Q, \\
S> & \left(\mathcal{A}_{0}^{T}\right)^{i-1} P_{1} \mathcal{A}_{0}^{i-1},
\end{aligned}
$$


hold for all $i \in\{1, \ldots, N\}$. Then, for any $\chi(0) \in \mathbb{R}^{2 n_{x}+n_{y}}$ and all $\bar{p} \in \widehat{\mathcal{P}}_{N, \eta}$, system (21) is MSS and the cost $J_{\mathrm{CONT}}(\chi(0))$ in (18) is upper bounded as follows

$$
J_{\mathrm{CONT}}(\chi(0)) \leq \max _{n \in\{1, \ldots, N+1\}} \chi(0)^{T} \bar{P}_{N}(n) \chi(0),
$$

for $\bar{P}_{N}(n)$ defined in (23).

Theorem 1 can be used directly to compute performance bounds on $J_{\mathrm{CONT}}(\chi(0))$ once $Q, N, \delta, \eta$ are fixed. These LMIs are different with respect to the ones found in the classical MJLS literature [21] since they involve the powers of $\mathcal{A}_{0}$, along with the summation $\bar{Q}_{N}(n)$ in order to yield tailored conditions for the SATI formulation. Besides, the upper bound defined by $\bar{P}_{N}(n)$ in (27) is also not standard, since it depends on the powers of $\mathcal{A}_{0}$ and the only variables of the problem defined by (24)-(26). Furthermore, the use of MJLS classic conditions such as the ones given in [23] is not possible, since the policies in the transition probability matrix (20) are not known a priori, but must respect $\bar{p} \in \widehat{\mathcal{P}}_{N, \eta}$.

When the original controller (2) has been designed to minimize the cost associated to a linear-quadratic regulator (LQR) problem in the absence of network, one may wonder, in view of Theorem 2, how this cost is impacted by the wireless channel. To answer this question, we concentrate on the case where the controller (2) is chosen as a state-feedback type, which minimizes a linear quadratic (LQ) cost. Specifically, we want to compare the SATI performance bound with the optimal cost given by the classic, that can be found by solving, for a given $x_{p}(0)$,

$$
J_{\mathrm{LQR}}\left(x_{p}(0)\right):=\min _{u} \sum_{t=0}^{\infty}\left[\begin{array}{c}
x_{p}(t) \\
u(t)
\end{array}\right]^{T}\left[\begin{array}{cc}
Q_{\mathrm{LQR}} & 0 \\
0 & R_{\mathrm{LQR}}
\end{array}\right]\left[\begin{array}{c}
x_{p}(t) \\
u(t)
\end{array}\right]
$$

for a given positive semi-definite matrix $Q_{\mathrm{LQR}}$ and a positive definite matrix $R_{\mathrm{LQR}}$. The solution of (28) is a state-feedback controller with the form,

$$
u(t)=K_{\mathrm{LQR}} x_{p}(t),
$$

that is associated with a discrete-time algebraic Riccati equation whose solution we denote by $P_{\mathrm{LQR}}$, see, for instance, [24]. In this context, we set $C_{p}=I$ in (1), as well as $A_{c}=0, B_{c}=0, C_{c}=0$, and $D_{c}=K_{\mathrm{LQR}}$ in (2). We also consider that the networked version of the state denoted by $\hat{x}_{p}(t)$ is generated by a zero-order-hold scheme between two successful transmissions, i.e., $C_{g}=I$ and $D_{g}=0$ in (4) and $\chi(t)=\left[x_{p}(t)^{T} \hat{x}(t-1)^{T}\right]^{T}$ in (6). In order to establish a comparison between the deterministic LQR cost in (28) and the upper bound of the SATI cost in (27), we present the following corollary.

Corollary 1: Given $\mu>0, N \in \mathbb{Z}_{>0}, \eta \in[0,1), \delta \in[0,1)$, and $x_{p}(0) \in \mathbb{R}^{n}$, we have that by setting the initial conditions of the problem (18) as $\hat{x}(-1)=x_{p}(0), \theta(0)=1$, and $Q$ in (18) as

$$
Q=\left[\begin{array}{cc}
Q_{\mathrm{LQR}} & 0 \\
0 & K_{\mathrm{LQR}}^{T} R_{\mathrm{LQR}} K_{\mathrm{LQR}}
\end{array}\right]
$$

we have that, if (24)-(26) and

$$
P_{1}<\frac{\mu}{2}\left[\begin{array}{cc}
P_{\mathrm{LQR}} & 0 \\
0 & P_{\mathrm{LQR}}
\end{array}\right]
$$

hold for symmetric positive definite matrices $P_{1}, P_{N+1}$, and $S$, then $J_{\mathrm{CONT}}(\chi(0)) \leq \mu J_{\mathrm{LQR}}\left(x_{p}(0)\right)$.

Corollary 1 provides us with the bound $J_{\mathrm{CONT}}(\chi(0)) \leq$ $\mu J_{\mathrm{LQR}}\left(x_{p}(0)\right)$, which implies that under the presence of the wireless network and the SATI conditions, the control cost is bounded by the LQR cost of the system without the network multiplied by the attenuation factor $\mu$. The matrix $Q$ in (29) is chosen so that the cost evaluated with (18) will correspond to $J_{\mathrm{LQR}}\left(x_{p}(0)\right)$ in the absence of the network. Note that by imposing the additional LMI (30) to (24)-(26), we are able to compare the SATI upper bound with the optimal LQR cost. We consider $\theta(0)=1$, i.e., a successful transmission is assumed to have occurred at $t=0$, which is required to simplify the bound in (27). Besides, as (30) is linear in $\mu$, the minimum attenuation $\mu^{*}$ can be found by minimizing $\mu$ over (24)-(26) and (30) by standard LMI solvers.

\section{Communication optimization}

Theorem 1 provides conditions on a set of transmission policies $\bar{p} \in \widehat{\mathcal{P}}_{N^{*}, \eta}$, which ensures the MSS of (7) and bounds on the control cost given in (18), respectively. We can then exploit this information to minimize the communication cost in (19). Let $\omega_{n}(\bar{p}):=\operatorname{Pr}(\theta(t)=n)$ for $n \in\{1, \ldots, N+1\}$ at the steady state of the Markov chain defined by the transition matrix $\Pi(\bar{p})$. The communication cost (19) can be evaluated as

$$
J_{\text {COMM }}(\bar{p})=p_{\max } \omega_{N+1}(\bar{p})+\sum_{n=1}^{N} \bar{p}_{n} \omega_{n}(\bar{p})
$$

where we can use the transition matrix $\Pi(\bar{p})$ to evaluate

$$
\omega_{n}(\bar{p})=\frac{\prod_{i=1}^{n-1} e\left(\bar{p}_{i}\right)}{\frac{1-\eta}{\delta}+\sum_{j=1}^{N} \prod_{i=1}^{j-1} e\left(\bar{p}_{i}\right)},
$$

for any $n\{1, \ldots, N\}$ and

$$
\omega_{N+1}(\bar{p})=\frac{\frac{1-\eta}{\delta}}{\frac{1-\eta}{\delta}+\sum_{j=1}^{N} \prod_{i=1}^{j-1} e\left(\bar{p}_{i}\right)} .
$$

Therefore, $J_{\mathrm{COMM}}(\bar{p})$ is a continuous and differentiable function w.r.t $\bar{p}$ and goal is to solve the following optimization problem.

$$
\begin{gathered}
\underset{\bar{p} \in \mathbb{R}^{N}}{\operatorname{minimize}} J_{\mathrm{COMM}}(\bar{p}), \\
\text { subject to } \prod_{i=1}^{N} e\left(\bar{p}_{n}\right) \leq 1-\eta^{*}, \\
0 \leq \bar{p}_{n} \leq p_{\max } .
\end{gathered}
$$

This is solved numerically by using the Lagrangian algorithm [25] as illustrated in the next section.

\section{ILluSTRATIVE EXAMPLES}

We consider the exactly discretized model of the unstable batch reactor given in [26]. Systems matrices $A$ and $B$, 
obtained with sampling period $T=50 \mathrm{~ms}$, are as follows

$$
\begin{aligned}
A_{p} & =\left[\begin{array}{rrrr}
1.0795 & -0.0045 & 0.2896 & -0.2367 \\
-0.0272 & 0.8101 & -0.0032 & 0.0323 \\
0.0447 & 0.1886 & 0.7317 & 0.2354 \\
0.0010 & 0.1888 & 0.0545 & 0.9115
\end{array}\right], \\
B_{p}^{T} & =\left[\begin{array}{rrrr}
0.0006 & 0.2567 & 0.0837 & 0.0837 \\
-0.0239 & 0.0002 & -0.1346 & -0.0046
\end{array}\right],
\end{aligned}
$$

and we assume that the states of system (1) can be measured, that is, $C_{p}=I$. We consider the topology described in (8)(9) that models the case in which the wireless network is in between the sensor and the controller, along with only state feedback controllers, and thus $A_{c}=0, B_{c}=0, C_{c}=0$. We calculate the controller by means of the LQR problem in (28) with $Q_{\mathrm{LQR}}=I$ and $R_{\mathrm{LQR}}=I$ resulting in the following state-feedback gain

$$
D_{c}=\left[\begin{array}{rrrr}
0.0153 & -0.8159 & -0.2394 & -0.7515 \\
2.3250 & 0.0801 & 1.6225 & -1.0657
\end{array}\right],
$$

and the solution $P_{\mathrm{LQR}}$ given by

$$
P_{\mathrm{LQR}}=\left[\begin{array}{crcc}
27.7052 & -0.2971 & 16.1405 & -13.6650 \\
-0.2971 & 3.3808 & 0.4508 & 2.1041 \\
16.1405 & 0.4508 & 11.9388 & -7.4574 \\
-13.6650 & 2.1041 & -7.4574 & 12.2756
\end{array}\right] .
$$

We now study the WNCS in (3), using a zero-order hold, i.e., $C_{g}=I$ and $D_{g}=0$. The wireless network is described by $c=0.9$ and $p_{\max }=1$ in (10) that leads to $\delta=1-$ $\exp (-0.9) \approx 0.6$.

For the first numerical study, we fix the attenuation parameter $\mu$ and investigate the behavior of the remaining SATI parameters. Figure 3 shows the minimum value of $\eta$ such that the conditions in Corollary 1 hold as a function of $N$ for $\mu \in\{10,30\}$. As expected, by restricting $\mu$, we have smaller feasible values of $N$ as $\mu$ decreases, i.e., taking $\mu=10$ will the feasible set of $N$ has a maximum value of $N=5$, whereas for $\mu=30$, it is possible to have feasible conditions up to $N=6$. On the other hand, it is necessary to increase the minimum values of $\eta$ for larger values of $N$, that is, we are increasing the energy spent on communication power in order to achieve feasibility for the chosen values of $\mu$.

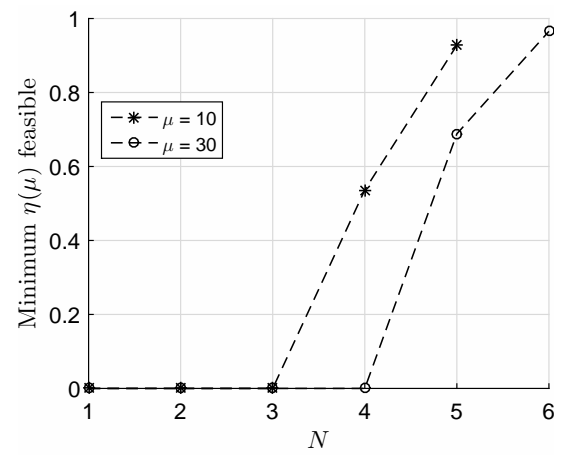

Fig. 3: The smallest feasible $\eta$ againt $N$ for $\delta=0.6$ and $\mu \in\{10,30\}$ obtained through Corollary 1 .

We next investigate the minimum communication cost possible, obtained numerically by running a Lagrangian algorithm for the minimum values of $\eta$ as a function of $N$ in Figure 3. In this context, Figure 4 shows the minimum communication costs $J_{\mathrm{COMM}}\left(\bar{p}^{*}\right)$ for a given $N$ and $\eta$ plotted in Figure 3. The costs in Figure 4 are decreasing up to $N=4$ for $\mu=10$, and $N=5$ for $\mu=30$. This decrease is expected since (19) will naturally reduce by decreasing the frequency of communication (as the minimum $\eta$ required is 0 for small values of $N$ ). However, after a certain range there is an increase in the communication cost due to a drastic increase in the minimum $\eta$ required for satisfying the control cost attenuation $\mu$ as seen in Figure 4. For this situation, we show in Table I the optimal power control policies for $\mu=10$ and $N=4$, as well as $\mu=30$ and $N=5$ (the minimum communication cost from Figure 4).

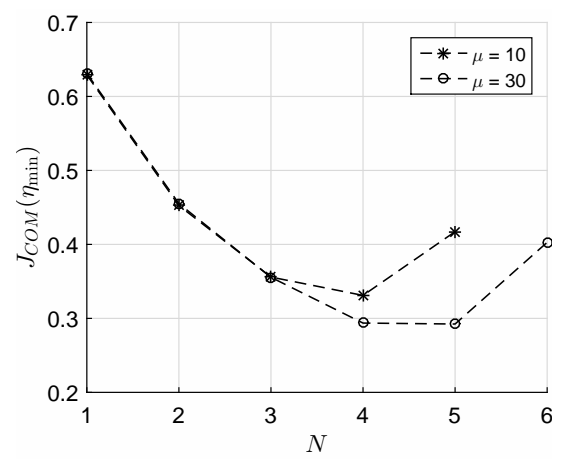

Fig. 4: Communication costs for the best feasible $\eta$.

TABLE I: Optimal power policies $\bar{p}_{\tau}^{*}$ for $\mu=10$ and $N=4$, as well as $\mu=30$ and $N=5$, of Figure 4 .

\begin{tabular}{||cccccccc||}
\hline$\mu$ & $N^{*}$ & $\eta^{*}$ & $\bar{p}_{1}^{*}$ & $\bar{p}_{2}^{*}$ & $\bar{p}_{3}^{*}$ & $\bar{p}_{4}^{*}$ & $\bar{p}_{5}^{*}$ \\
\hline \hline 10 & 4 & 0.54 & 0 & 0 & 0.24 & 0.58 & - \\
\hline 30 & 5 & 0.68 & 0 & 0 & 0.14 & 0.42 & 0.75 \\
\hline
\end{tabular}

We now perform a comparison with the SATI based approach and event-triggered control (ETC). For that, we consider a modified version of the ETC rule presented in [27] described as follows. When $\|\hat{x}(t)-x(t)\|>\gamma\|x(t)\|$, we set $p(t)=p_{\max }$ and otherwise $p(t)=0$. We set $c=10$ and $p_{\max }=1$ in (10) resulting in $\delta=0.9999$, which is the probability of successful transmission when $p(t)=p_{\max }$. We compute via simulations the expected control and communication costs applying this ETC rule with each point of the plot corresponding to certain values of $\gamma \in\{0.10,0.38,0.66,0.94,1.22\}$ (for $\gamma>1.22$, the closedloop system becomes unstable). We compare this with a power control based on SATI rules considering the pairs $(N, \eta) \in$ $\{(1,0.955),(2,0.960),(3,0.965),(4,0.970),(5,0.975)\}$, where the power control policy $\bar{p}$ is calculated through (34) for each pair. The resulting pairs $\mathbb{E}\left(J_{\mathrm{COM}}\right) \times \mathbb{E}\left(J_{\mathrm{CON}}\right)$ for ETC and SATI are shown in Figure 5 for a Monte Carlo simulation of 4000 rounds with each round having random initial conditions $x_{p}(0)$ sampled from a Gaussian distribution. We assume that $\hat{x}(-1)=x_{p}(0)$ for the ETC and SATI 
control. We see that for a fixed control cost, the optimized power control using the SATI approach is able to provide a smaller communication cost compared to the ETC for the considered set of simulations and associated parameters.

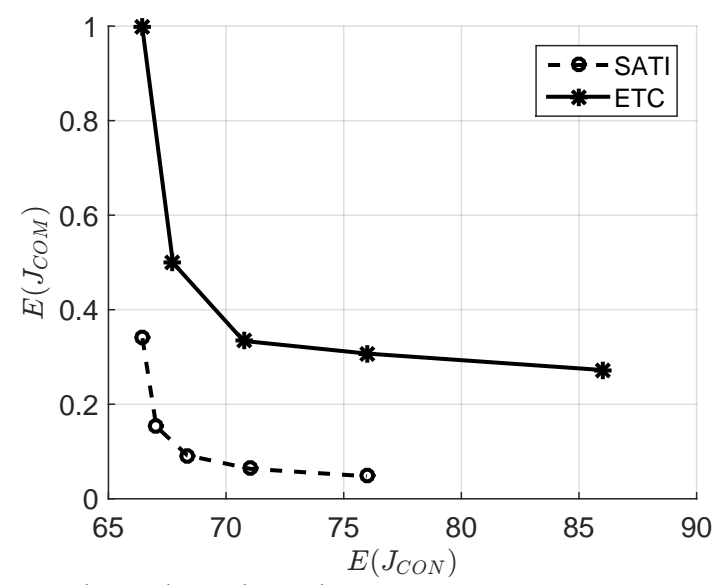

Fig. 5: $\mathbb{E}\left(J_{\mathrm{COM}}\right) \times \mathbb{E}\left(J_{\mathrm{CON}}\right)$ with each point corresponding to a certain $\gamma$ for ETC, and pair $(N, \eta)$ (with optimal $\bar{p}$ ) for SATI.

\section{CONCLUSION}

We have studied discrete-time linear systems in which the communication with the controller occurs over a stochastic wireless channel. The wireless communication system is such that the probability of packet loss depends on the power of the transmitting antenna. We consider a time-triggered power control policy, i.e., the signal power is designed to be a function of the clock-state $\tau(t)$. We introduce the notion of $(\eta, \delta)$ SATI which is an interpretation of the traditional MATI in the stochastic setting, where $\eta$ denotes the cumulative probability of a successful transmission within the $(\eta, \delta)$-SATI and $\delta$ denotes the instantaneous communication success probability after the $(\eta, \delta)$-SATI has passed. Under the assumption that the system with perfect communication is stabilizable, we provide conditions on the $(\eta, \delta)$-SATI in terms of matrix inequalities which imply MSS and certain performance bounds. We have then presented algorithm using which a communication engineer can optimize the transmission power control policy in order to minimize the average expected communication energy cost. Our numerical study demonstrates how control engineers and communication engineers may exploit the provided results in order to design energy-efficient policies for a wireless NCS.

\section{APPENDIX}

Before providing the proofs of Theorem 1 and Corollary 1, we first introduce the following auxiliary result.

Lemma 1: Consider that $0 \leq \eta<1$ and define

$$
F(n):=\sum_{i=n}^{N}\left(1-e\left(\bar{p}_{i}\right)\right) \prod_{j=n}^{i-1} e\left(\bar{p}_{j}\right), n \in\{1, \ldots, N\} .
$$

We have $F(1)=\eta$ and $F(n) \leq \eta$ for all $n \in\{1, \ldots, N\}$.

Proof: Through simple manipulation on (35), we get that,

$$
1-F(n)=e\left(\bar{p}_{n}\right)(1-F(n+1))
$$

holds for any for any $n<N$. We know that $F(N)=1-e\left(\bar{p}_{N}\right)$ by definition. Therefore we get through recursion and (15) that

$$
1-F(1)=\prod_{i=1}^{N} e\left(\bar{p}_{i}\right)=1-\eta .
$$

Since $\eta \neq 1$, then $0<e\left(\bar{p}_{j}\right) \leq 1$, and thus $0<1-F(n) \leq 1$ for any $n$ due to (36) and $0<1-F(N) \leq 1$. It follows that $1-F(n)=e\left(\bar{p}_{n}\right)(1-F(n+1)) \leq 1-\bar{F}(n+1)$ for all $n$ (equality arises if $e\left(\bar{p}_{j}\right)=1$ ). Therefore $F(n) \geq F(n+1)$, and particularly, $\eta=F(1) \geq F(n)$ for all $n \in\{1, \ldots, N\}$.

In order to prove Theorem 1 , we set, for $m \in\{2, \ldots, N-1\}$,

$$
\Gamma(m):=\mathcal{A}_{0}^{T}\left[\left(1-e\left(\bar{p}_{m}\right)\right) P_{1}+e\left(\bar{p}_{m}\right) \Gamma(m+1)\right] \mathcal{A}_{0}+Q,
$$

and $\Gamma(N):=\mathcal{A}_{0}^{T}\left[\left(1-e\left(\bar{p}_{N}\right)\right) P_{1}+e\left(\bar{p}_{N}\right) P_{N+1}\right] \mathcal{A}_{0}+Q$.

Proof of Theorem 1: For the stability part, by setting $Q=0$ in (24)-(25), we directly get the stability condition in [28], and thus (21) is MSS for all $\bar{p} \in \widehat{\mathcal{P}}_{N, \eta}$. Considering the performance part, the exact values of (18) for MJLS MSS systems such that $\bar{p} \in \widehat{\mathcal{P}}_{N, \eta}$ can be calculated from Lemma A.1 of [23]. This is obtained by solving a set of coupled Lyapunov equations whose solutions we denote by $P_{n}^{*}(\bar{p}) \geq 0$, $n \in\{1, \ldots, N+1\}$ for all $\bar{p} \in \widehat{\mathcal{P}}_{N, \eta}$. Recalling that $\left(\prod_{j=n}^{i-1} e\left(\bar{p}_{j}\right)\right) \leq 1$ for any $n \in\{1, \ldots, N\}, i \in\{2, \ldots, N\}$, we have that

$$
\bar{Q}_{N}(2) \geq \sum_{i=2}^{N}\left(\mathcal{A}_{0}^{T}\right)^{i-2} Q \mathcal{A}_{0}^{i-2} \prod_{j=1}^{i-1} e\left(\bar{p}_{j}\right) .
$$

Besides, by multiplying (26) by $\left(1-e_{i}\right) \prod_{j=1}^{i-1} e_{j}$, summing it up for all $i \in\{1, \ldots, N\}$, and considering Lemma 1, we get that

$$
\eta S \geq \sum_{i=1}^{N}\left(1-e_{i}\right) \prod_{j=1}^{i-1} e_{j}\left(\mathcal{A}_{0}^{T}\right)^{i-1} P_{1} \mathcal{A}_{0}^{i-1} .
$$

Considering (39) and (40), and after some tedious but straightforward manipulations, we can rewrite (24) as follows

$$
\Phi(1):=P_{1}-\mathcal{A}_{1}^{T}\left[\left(1-e\left(\bar{p}_{1}\right)\right) P_{1}+e\left(\bar{p}_{1}\right) \Gamma(2)\right] \mathcal{A}_{1}+Q>0,
$$

for $\Gamma(2)$ defined in (38). The idea is to apply a suitable perturbation $\epsilon_{2}$ in (41), that depends on the eigenvalues of $\Phi(1)$ and $\mathcal{A}_{1}$, such that $P_{1}-\mathcal{A}_{1}^{T}\left[\left(1-e\left(\bar{p}_{1}\right)\right) P_{1}+e\left(\bar{p}_{1}\right)\left(\Gamma(2)+I \epsilon_{2}\right)\right] \mathcal{A}_{1}+Q>0$ still holds. We define $P_{2}(\bar{p})>0, \Gamma(2)+I \epsilon_{2}>P_{2}(\bar{p})>\Gamma(2)$ and obtain that $P_{1}>\mathcal{A}_{1}^{T}\left[\left(1-e\left(\bar{p}_{1}\right)\right) P_{1}+e\left(\bar{p}_{1}\right) P_{2}(\bar{p})\right] \mathcal{A}_{1}+Q>$ 0 also holds. By using the recursive equations in (38) and the previous similar reasoning, we are able to write the following inequalities,

$$
\begin{aligned}
P_{1} & >\mathcal{A}_{1}^{T}\left[\left(1-e\left(\bar{p}_{1}\right)\right) P_{1}+e\left(\bar{p}_{1}\right) P_{2}(\bar{p})\right] \mathcal{A}_{1}+Q, \\
P_{n}(\bar{p}) & >\mathcal{A}_{0}^{T}\left[\left(1-e\left(\bar{p}_{n}\right)\right) P_{1}+e\left(\bar{p}_{n}\right) P_{n+1}(\bar{p})\right] \mathcal{A}_{0}+Q,
\end{aligned}
$$

for $n \in\{2, \ldots, N\}, \bar{p} \in \widehat{\mathcal{P}}_{N, \eta}$, along with (25). Summoning Corollary 2.7 and Proposition 3.20 from [21], we get that $P_{1}^{*}(\bar{p})<P_{1}$ and $P_{N+1}^{*}(\bar{p})<P_{N+1}$, for all $\bar{p} \in \widehat{\mathcal{P}}_{N, \eta}$. From 
Lemma A.1 of [23], we have that

$J_{\mathrm{CONT}}\left(\chi_{0}\right)=\sum_{n=1}^{N} \nu_{n} \chi_{0}^{T} P_{n}^{*}(\bar{p}) \chi_{0} \leq \max _{n \in\{1, \ldots, N+1\}} \chi_{0}^{T} P_{n}^{*}(\bar{p}) \chi_{0}$

where $\nu_{i}=\operatorname{Pr}(\theta(0)=i)$. The inequality (44) results from the fact that $0 \leq \nu_{i} \leq 1$ and $\chi_{0}^{T} P_{i}^{*}(\bar{p}) \chi_{0} \geq 0, i \in$ $\{1, \ldots, N+1\}$. From the previous discussion, it is direct that $P_{1}^{*}(\bar{p})<\bar{P}_{N}(1)=P_{1}$ and $P_{N+1}^{*}(\bar{p})<\bar{P}_{N}(N+1)=P_{N+1}$, thus it remains to show the bounds for $n \in\{2, \ldots, N\}$. Through Equation A.1 in [23], we can write any $P_{n}^{*}(\bar{p})$, $n \in\{2, \ldots, N\}$, as follows,

$$
\begin{aligned}
& P_{n}^{*}(\bar{p})=\prod_{i=n}^{N} e\left(\bar{p}_{i}\right)\left(\mathcal{A}_{0}^{T}\right)^{N+1-n} P_{N+1}^{*}(\bar{p})\left(\mathcal{A}_{0}\right)^{N+1-n} \\
& +\sum_{i=n}^{N}\left(1-e\left(\bar{p}_{i}\right)\right) \prod_{j=n}^{i-1} e\left(\bar{p}_{j}\right)\left(\mathcal{A}_{0}^{T}\right)^{i-n+1} P_{1}^{*}(\bar{p}) \mathcal{A}_{0}^{i-n+1} \\
& \quad+\sum_{i=n}^{N}\left(\mathcal{A}_{0}^{T}\right)^{i-n} Q \mathcal{A}_{0}^{i-n} \prod_{j=n}^{i-1} e\left(\bar{p}_{j}\right)
\end{aligned}
$$

We now show that each term in (45) is bounded: (i) Since $0<\prod_{i=n}^{N} e\left(\bar{p}_{i}\right) \leq 1$, and $P_{N+1}^{*}(\bar{p})<P_{N+1}$, we get that $\prod_{i=n}^{N} e\left(\bar{p}_{i}\right)\left(\mathcal{A}_{0}^{T}\right)^{N+1-n} P_{N+1}^{*}(\bar{p})\left(\mathcal{A}_{0}\right)^{N+1-n} \leq$ $\left(\mathcal{A}_{0}^{T}\right)^{N+1-n} P_{N+1}\left(\mathcal{A}_{0}\right)^{N+1-n}, n \in\{2, \ldots, N\}$; (ii) By changing the index in (26), multiplying the resulting equation by $\left(1-e\left(\bar{p}_{i}\right)\right) \prod_{j=n}^{i-1} e\left(\bar{p}_{j}\right)$, summing it up for $i \in\{n, \ldots, N\}$, and considering Lemma 1 , we get that $\sum_{i=n}^{N}\left(1-e\left(\bar{p}_{i}\right)\right) \prod_{j=n}^{i-1} e\left(\bar{p}_{j}\right)\left(\mathcal{A}_{0}^{T}\right)^{i-n+1} P_{1}^{*}(\bar{p}) \mathcal{A}_{0}^{i-n+1} \leq$ $\sum_{i=n}^{N}\left(1-e\left(\bar{p}_{i}\right)\right) \prod_{j=n}^{i-1} e\left(\bar{p}_{j}\right)\left(\mathcal{A}_{0}^{T}\right)^{i-n+1} P_{1} \mathcal{A}_{0}^{i-n+1} \leq$ $\sum_{i=n}^{N}\left(1-e\left(\bar{p}_{i}\right)\right) \prod_{j=n}^{i-1} e\left(\bar{p}_{j}\right) S \leq \eta S$, for all $n \in\{2, \ldots, N\}$, $\bar{p} \in \widehat{\mathcal{P}}_{N, \eta}$; (iii) Since $0<\prod_{j=n}^{i-1} e\left(\bar{p}_{j}\right) \leq 1$, we get that $\sum_{i=n}^{N}\left(\mathcal{A}_{0}^{T}\right)^{i-n} Q \mathcal{A}_{0}^{i-n} \prod_{j=n}^{i-1} e\left(\bar{p}_{j}\right) \leq$ $\sum_{i=n}^{N}\left(\mathcal{A}_{0}^{T}\right)^{i-n} Q \mathcal{A}_{0}^{i-n} \prod_{j=n}^{i-1}$ for all $n \in\{2, \ldots, N\}$, $\bar{p} \in \widehat{\mathcal{P}}_{N, \eta}$. Thus, $P_{n}^{*}(\bar{p}) \leq \bar{P}_{N}(n)$ for all $n \in\{1, \ldots, N+1\}$ and $\bar{p} \in \widehat{\mathcal{P}}_{N, \eta}$, and by considering (44), the claim follows.

Proof of Corollary 1: By taking $\hat{x}(-1)=x_{p}(0)$ and $\theta(0)=1$, and considering the zero-order-hold strategy given in (4), we have, by multiplying (30) by $\chi(0)^{T}$ from the left hand side and $\chi(0)$ to the right hand side, that $\chi(0)^{T} P_{1} \chi(0) \leq \mu x_{p}(0)^{T} P_{\mathrm{LQR}} x_{p}(0)=J_{\mathrm{LQR}}\left(x_{p}(0)\right)$. Note that whenever we consider that $\theta(0)=1$, the optimal MJLS cost in (44) becomes $J_{\mathrm{CONT}}(\chi(0), \bar{p})=\chi(0)^{T} P_{1}^{*}(\bar{p}) \chi(0)$. The claim follows recalling that $P_{1}^{*}(\bar{p})<P_{1}$ for all $\bar{p} \in \widehat{\mathcal{P}}_{N, \eta}$ from Theorem 1.

\section{REFERENCES}

[1] J. Wu, S. Rangan, and H. Zhang. Green communications: theoretical fundamentals, algorithms, and applications. CRC Press, 2016.

[2] T. Rault, A. Bouabdallah, and Y. Challal. Energy efficiency in wireless sensor networks: A top-down survey. Computer Networks, 67:104-122, 2014.

[3] D. Goodman and N. Mandayam. Power control for wireless data. IEEE Personal Communications, 7(2):48-54, 2000.

[4] V.S. Varma, S. Lasaulce, M. Debbah, and S.E. Elayoubi. An energyefficient framework for the analysis of mimo slow fading channels. IEEE Transactions on Signal Processing, 61(10):2647-2659, 2013.
[5] W.P.M.H. Heemels and N. van de Wouw. Stability and stabilization of networked control systems. In Networked Control Systems, volume Volume 406 of the series Lecture Notes in Control and Information Sciences, pages 203-253. Springer, 2010.

[6] V.S. Varma and R. Postoyan. Energy efficient time-triggered control over wireless sensor/actuator networks. In IEEE Conference on Decision and Control, Las Vegas, U.S.A., 2016.

[7] N.C. De Castro, C.C. De Wit, and K.H. Johansson. On energy-aware communication and control co-design in wireless networked control systems. In 2nd IFAC Workshop on Distributed Estimation and Control in Networked Systems, Annecy, France, pages 49-54, 2010.

[8] M. Rabi and K.H. Johansson. Event-triggered strategies for industrial control over wireless networks. In Proceedings of the 4th annual international conference on wireless internet, page 34. ICST (Institute for Computer Sciences, Social-Informatics and Telecommunications Engineering), 2008.

[9] X. Liu and A. Goldsmith. Wireless medium access control in networked control systems. In American Control Conference, 2004. Proceedings of the 2004, volume 4, pages 3605-3610. IEEE, 2004.

[10] A. Molin and S. Hirche. On lqg joint optimal scheduling and control under communication constraints. In Decision and Control, 2009 held jointly with the 2009 28th Chinese Control Conference. CDC/CCC 2009. Proceedings of the 48th IEEE Conference on, pages 5832-5838. IEEE, 2009.

[11] K. Gatsis, A. Ribeiro, and G.J. Pappas. Optimal power management in wireless control systems. IEEE Transactions on Automatic Control, 59(6):1495-1510, 2014.

[12] Daniel E Quevedo, Anders Ahlén, Alex S Leong, and Subhrakanti Dey. On kalman filtering over fading wireless channels with controlled transmission powers. Automatica, 48(7):1306-1316, 2012.

[13] L.A. Montestruque and P. Antsaklis. Stability of model-based networked control systems with time-varying transmission times. IEEE Transactions on Automatic Control, 49(9):1562-1572, 2004.

[14] L. Xie and L. Xie. Stabilizing sampled-data linear systems with markovian packet losses and random sampling. IFAC Proceedings Volumes, 41(2):599-604, 2008.

[15] V.S. Varma, R. Postoyan, I-C. Morărescu, and J. Daafouz. Stochastic maximum allowable transmission intervals for the stability of linear wireless networked control systems. In Decision and Control (CDC), 2017 IEEE 56th Annual Conference on, pages 6634-6639. IEEE, 2017.

[16] L. Schenato. To zero or to hold control inputs with lossy links? IEEE Transactions on Automatic Control, 54(5):1093-1099, 2009.

[17] L.H. Ozarow, S. Shamai, and A.D. Wyner. Information theoretic considerations for cellular mobile radio. IEEE transactions on Vehicular Technology, 43(2):359-378, 1994.

[18] S. R. Theodore. Wireless communications: principles and practice, volume 2. Prentice Hall PTR New Jersey, 1996.

[19] D. Nešić and A.R. Teel. Input-output stability properties of networked control systems. IEEE Trans. on Aut. Control, 49:1650-1667, 2004.

[20] R. Postoyan and D. Nešić. Time-triggered control of nonlinear discretetime systems. In IEEE Conference on Decision and Control, Las Vegas, U.S.A., pages 6814-6819, 2016.

[21] O.L.V. Costa, M.D. Fragoso, and R.P. Marques. Discrete-Time Markov Jump Linear Systems. Springer, 2005.

[22] E. Hossain, V.K. Bhargava, and G.P. Fettweis. Green radio communication networks. Cambridge University Press, 2012.

[23] João B. do Val, José C. Geromel, and Alim P. C. Gonçalves. The $\mathrm{H}_{2}$ control for jump linear systems: cluster observations of the Markov state. Automatica, 38(2), 2002.

[24] K. Zhou, J.C. Doyle, and K. Glover. Robust and optimal control, volume 40. Prentice hall New Jersey, 1996.

[25] D.P. Bertsekas. Constrained optimization and Lagrange multiplier methods. Academic Press, 1982.

[26] G. C. Walsh, H. Ye, and G. Bushnell. Stability analysis of networked control systems. IEEE Transactions Control Systems Technology, 10(3):438-446, 2002.

[27] W. P. M. H. Heemels, M. C. F. Donkers, and A. R. Teel. Periodic eventtriggered control for linear systems. IEEE Transactions on Automatic Control, 58(4):847-861, April 2013.

[28] A.M.D Oliveira, V.S. Varma, R. Postoyan, C. Morarescu, J. Daafouz, and O.L.V. Costa. Co-design of state-feedback controllers and stochastic transmission policies for linear wireless networked control systems. In IFAC Conference on Analysis and Design of Hybrid Systems (ADHS). IFAC, 2018. 